\title{
Decentralized control of vehicle formations
}

\author{
G. Lafferriere*, ${ }^{*}$ A. Williams ${ }^{1}$, J. Caughman, J.J.P. Veerman \\ Department of Mathematics and Statistics, Portland State University, Portland, OR 97207-0751, USA
}

Received 9 July 2004; received in revised form 4 December 2004; accepted 5 February 2005

Available online 17 March 2005

\begin{abstract}
This paper investigates a method for decentralized stabilization of vehicle formations using techniques from algebraic graph theory. The vehicles exchange information according to a pre-specified communication digraph, $G$. A feedback control is designed using relative information between a vehicle and its in-neighbors in $G$. We prove that a necessary and sufficient condition for an appropriate decentralized linear stabilizing feedback to exist is that $G$ has a rooted directed spanning tree. We show the direct relationship between the rate of convergence to formation and the eigenvalues of the (directed) Laplacian of $G$. Various special situations are discussed, including symmetric communication graphs and formations with leaders. Several numerical simulations are used to illustrate the results.
\end{abstract}

(C) 2005 Elsevier B.V. All rights reserved.

Keywords: Formation stability; Graph Laplacian; Algebraic graph theory; Decentralized control; Rooted directed spanning tree

\section{Introduction}

There is growing interest in the decentralized control of multiple autonomous vehicles due, in part, to a number of new important applications. These include the coordinated control of minisatellites, drone planes, and underwater vehicles

\footnotetext{
* Corresponding author. Tel.: +1 5037253662 ; fax: +15037253661 .

E-mail addresses: gerardoL@pdx.edu (G. Lafferriere), ancaw@pdx.edu (A. Williams), caughman@pdx.edu (J. Caughman), veerman@pdx.edu (J.J.P. Veerman).

${ }^{1}$ G. Lafferriere and A. Williams were supported in part by a grant from Honeywell, Inc. G. Lafferriere was supported in part by NSF Grant DMS-0408334 and by a Career Support grant from Portland State University.
}

$[4,5,7,9-13,20,22-25,29]$. One of the main goals is to achieve a coordinated objective while using only relative information concerning positions and velocity. The objective investigated in this paper is that of attaining a moving formation. That is, the goal of the vehicles is to achieve and maintain pre-specified relative positions and orientations with respect to each other. Each vehicle is provided information only from a subset of the group. The specific subset is given through the set of "neighbors" in the communication digraph. This digraph need not be related in any way to the actual position of the vehicles.

The feedback scheme investigated here was proposed by Fax and Murray in [5] and is inspired by the motion of aggregates of individuals in nature. Flocks of birds and schools of fish achieve coordinated 
motions without the use of a central controlling mechanism [16]. Another simple model is proposed in [27] that captures the observed motions of self-driven particles. Both of these models employ feedback laws in which the motions of nearest-neighbors are averaged. The notion of a communication digraph is introduced in [4], and an averaging feedback law is proposed based on the flow of information. The authors of [23-25] investigate the motions of vehicles modelled as double integrators. Their goal is for the vehicles to achieve a common velocity while avoiding collisions. The control laws involve graph Laplacians for an associated undirected (neighborhood) graph but also nonlinear terms resulting from artificial potential functions. Rather than reaching for a predetermined "formation", the vehicles converge to an equilibrium formation that minimizes all individual potentials. They also extend their results to the case of switching neighborhood graphs. Refs. $[11,20]$ contain a special case of the results presented here (among other results). In both references the communication graph is the undirected cycle. In [11] the vehicles are modelled directly as double integrators. In [20] a more complete, nonlinear model is introduced and then force and torque feedback is used to linearize the model before applying the graph-theoretic techniques.

Another area of research which is closely related to the problems and techniques discussed in this paper is that of consensus seeking by autonomous agents $[1,15,17-19,21]$. We say that agents $1, \ldots, n$ achieve consensus if their associated variables $x_{1}, \ldots, x_{n}$ converge to a common value (or, more generally, if $f\left(x_{1}\right), \ldots, f\left(x_{n}\right)$ converge to a common value for some given function $f$ ). The vector $x$ of variables satisfies a differential (difference) equation $\dot{x}=-L x(t)$ $(x(t+1)=-L x(t))$ where $L$ is (essentially) a weighted Laplacian of a digraph (the "communication" digraph). In [19,21] it is shown that a necessary and sufficient condition for achieving consensus is that the communication digraph admits a directed spanning tree. In $[17,18]$ the authors only prove sufficiency and assume that the graph is either undirected [18] or strongly connected [17]. Refs. [17,19,21] show that under certain conditions consensus can be achieved even when the communication graph switches over time. Control to formation is, in a way, a consensus problem since in order to reach a stable formation the vehicles must achieve, among other things, the same velocity. On the other hand, the vehicle dynamics of the formation problem are, in effect, governed by second-order equations.

In this paper we prove that the same condition of the communication graph above is necessary and sufficient for the existence of certain decentralized feedback laws that control the vehicles to arbitrary formations. We also provide a relationship between the rate of convergence to formation and the eigenvalues of the (directed) graph Laplacian. We demonstrate how, for a fixed feedback gain matrix, convergence can be improved by choosing alternative communication digraphs. Finally, we explore some special cases in more detail and illustrate the results with numerical examples.

We point out that the result of Proposition 3.4 below, already appears in $[19,21]$. Here we offer a short, purely graph-theoretic proof.

The paper is organized as follows. In Section 2 we set up the basic model. The relevant graph theoretic definitions and results are collected in Section 3. The main results are proved in Section 4, and illustrative examples are given in Section 5. In Section 6 we discuss two special cases-bidirectional communications and formations with leaders-in which additional refinements can be made. Various motions of the formation are illustrated in Section 7.

\section{Model}

We assume we are given $N$ vehicles with the same dynamics

$\dot{x}_{i}=A_{\mathrm{veh}} x_{i}+B_{\mathrm{veh}} u_{i}, \quad i=1, \ldots, N, \quad x_{i} \in \mathbb{R}^{2 n}$,

where the entries of $x_{i}$ represent $n$ configuration variables for vehicle $i$ and their derivatives, and the $u_{i}$ represent control inputs. For simplicity we will assume further that the matrices $A_{\text {veh }}$ and $B_{\text {veh }}$ have the form

$$
\begin{aligned}
& A_{\text {veh }}=\operatorname{diag}\left(\left(\begin{array}{cc}
0 & 1 \\
a_{21}^{1} & a_{22}^{1}
\end{array}\right), \ldots,\left(\begin{array}{cc}
0 & 1 \\
a_{21}^{n} & a_{22}^{n}
\end{array}\right)\right), \\
& B=I_{n} \otimes\left(\begin{array}{l}
0 \\
1
\end{array}\right)
\end{aligned}
$$

(where $\otimes$ denotes the Kronecker product). This corresponds to the individual configuration variables being decoupled and the acceleration of each such variable 
being controlled separately. These conditions may be relaxed. We will refer to the odd-numbered entries of $x=\left(x_{1}, \ldots, x_{N}\right)^{\mathrm{T}}$ as position-like variables and to the even-numbered entries as velocity-like variables. We will use the notation $x_{p}=\left(\left(x_{p}\right)_{1}, \ldots,\left(x_{p}\right)_{N}\right)^{\mathrm{T}}, x_{v}=$ $\left(\left(x_{v}\right)_{1}, \ldots,\left(x_{v}\right)_{N}\right)^{\mathrm{T}}$ to denote the vectors of positionlike and velocity-like variables, so $x=x_{p} \otimes\left(\begin{array}{l}1 \\ 0\end{array}\right)+$ $x_{v} \otimes\left(\begin{array}{l}0 \\ 1\end{array}\right)$.

Definition 2.1. A formation is a vector

$h=h_{p} \otimes\left(\begin{array}{l}1 \\ 0\end{array}\right) \in \mathbb{R}^{2 n N}$

(where $\otimes$ denotes the Kronecker product). The $N$ vehicles are in formation $h$ at time $t$ if there are vectors $q, w \in \mathbb{R}^{n}$ such that $\left(x_{p}\right)_{i}(t)-\left(h_{p}\right)_{i}=q$ and $\left(x_{v}\right)_{i}(t)=w$, for $i=1, \ldots, N$. The vehicles converge to formation $h$ if there exist $\mathbb{R}^{n}$-valued functions $q(\cdot), w(\cdot)$ such that $\left(x_{p}\right)_{i}(t)-\left(h_{p}\right)_{i}-q(t) \rightarrow 0$ and $\left(x_{v}\right)_{i}(t)-w(t) \rightarrow 0$, as $t \rightarrow \infty$, for $i=1, \ldots, N$ (where $x_{p}$ and $x_{v}$ are as indicated above).

Fig. 1 illustrates the interpretation of the vectors in the definition.

For more convenient mathematical treatment we introduce the following space associated with each formation vector.

Definition 2.2. Let $h=h_{p} \otimes\left(\begin{array}{l}1 \\ 0\end{array}\right) \in \mathbb{R}^{2 n N}$, let $\mathbf{1}_{N}$ denote the all ones vector of size $N$, and let $\mathbf{e}_{j}, j=$ $1, \ldots, 2 n$ denote the standard basis vectors in $\mathbb{R}^{2 n}$. Set $W=\left\{\mathbf{1}_{N} \otimes \mathbf{e}_{j}: j=1, \ldots, 2 n\right\}$. We define the $h$-formation space $\mathscr{F}_{h}$ by

$\mathscr{F}_{h}=h+\operatorname{span} W=\left\{x \mid \exists \gamma \in \mathbb{R}^{2 n}: x-h=\mathbf{1}_{N} \otimes \gamma\right\}$.

Notice that $x-h=\mathbf{1}_{N} \otimes \gamma$ is equivalent to $\left(x_{p}\right)_{i}-$ $\left(h_{p}\right)_{i}=q$ and $\left(x_{v}\right)_{i}=w$ where $\gamma=q \otimes\left(\begin{array}{l}1 \\ 0\end{array}\right)+w \otimes\left(\begin{array}{l}0 \\ 1\end{array}\right)$.

With this definition $x$ is in formation $h$ if and only if $x \in \mathscr{F}_{h}$ and $x(t)$ converges to formation $h$ if and only if the distance from $x(t)$ to the space $\mathscr{F}_{h}$ tends to zero as $t \rightarrow \infty$.

To complete the model we are also given a graph $G$ which captures the communication links between vehicles (see next section for precise definitions of graph theoretic concepts). Each vertex represents a vehicle

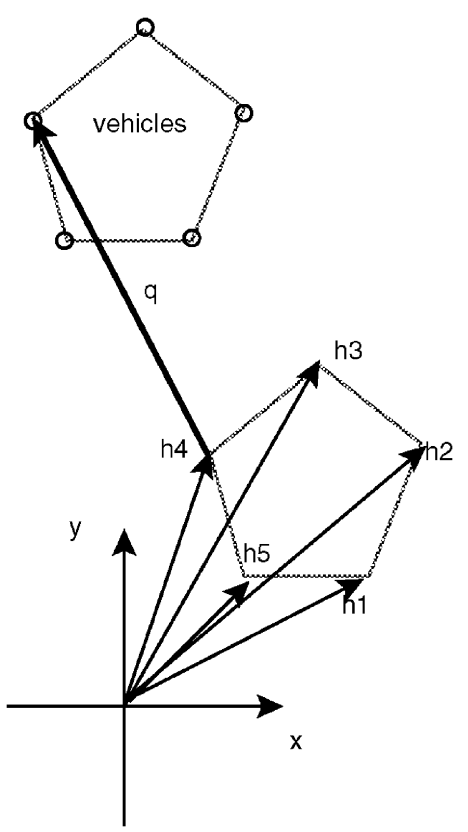

Fig. 1. Vehicles in formation.

and there is a directed edge from one vertex to another if there is a communication link sending information from the first vehicle to the second. The second vehicle would then be able to use this information in a feedback formula to adjust its own state. We say that the first vehicle is a neighbor of the second. For each vehicle $i, \mathbb{J}_{i}$ denotes the set of its neighbors. In this model, the decentralized nature of the feedback control law is captured by allowing each vehicle to use only relative information about its neighbors. More precisely, we are interested only in controls $u_{i}$ which are functions of $x_{j}-x_{i}$ and $h_{j}-h_{i}$ for each $j \in \mathbb{J}_{i}$. Note, however, that this still requires all vehicles to agree on a global reference frame with respect to which the differences $x_{j}-x_{i}$ can be described.

A natural way to combine the relative information (as done in [5]) is to define output functions $y_{i}$ computed from an average of the relative displacements (and velocities) of the neighboring vehicles as follows:

$$
\begin{gathered}
y_{i}=\left(x_{i}-h_{i}\right)-\frac{1}{\left|\mathbb{J}_{i}\right|} \sum_{j \in \unlhd_{i}}\left(x_{j}-h_{j}\right), \\
i=1, \ldots, N,
\end{gathered}
$$


where $\left|\mathbb{J}_{i}\right|$ indicates the number of neighbors to vehicle $i$. However, we will make a slight modification here to allow for the possibility that a vehicle might receive no information and so the corresponding vertex in the graph would have no neighbors. This situation occurs naturally if one of the vehicles is a designated leader around whose motions the others should adjust theirs. We will define the output functions $z_{i}$ by

$$
\begin{gathered}
z_{i}=\sum_{j \in \unlhd_{i}}\left(\left(x_{i}-h_{i}\right)-\left(x_{j}-h_{j}\right)\right), \\
\quad i=1, \ldots, N .
\end{gathered}
$$

As a result, the corresponding output vector $z$ can be written as $z=L(x-h)$ where $L=L_{G} \otimes I_{2 n}$ and $L_{G}$ is the (directed) Laplacian matrix of the communication graph $G$ (see Section 3).

Collecting the equations for all the vehicles into a single system we obtain

$\dot{x}=A x+B u$,

$z=L(x-h)$,

with $A=I_{N} \otimes A_{\mathrm{veh}}, B=I_{N} \otimes B_{\mathrm{veh}}$.

We are interested in studying the existence of feedback matrices $F$ such that the solutions to

$\dot{x}=A x+B F L(x-h)$

converge to formation $h$. This is a stabilization problem involving output feedback. In order to take advantage of the block structure of the matrices $A, B$, and $L$ we will restrict our study to matrices $F$ of the form $F=I_{N} \otimes F_{\text {veh }}$ (a particular "decentralized" control law where the same feedback law applies to all vehicles). In this case, we can write Eq. (3) as follows:

$\dot{x}=I_{N} \otimes A_{\mathrm{veh}} x+L_{G} \otimes B_{\mathrm{veh}} F_{\mathrm{veh}}(x-h)$.

The main purpose of the paper is to show the relationship between existence of feedback matrices $F_{\text {veh }}$ that guarantee convergence to formation and properties of the communication graph $G$.

Even before a more precise definition of $L_{G}$ we can show how its eigenvalues play a central role. Let $U$ be a matrix such that $\widetilde{L}_{G}=U^{-1} L_{G} U$ is upper triangular. In particular, the eigenvalues of $L_{G}$ are the diagonal entries of $\widetilde{L}_{G}$. A direct calculation using the special form of $A, B$, and $F$ gives

$$
\begin{gathered}
\left(U^{-1} \otimes I_{2 n}\right)(A+B F L)\left(U \otimes I_{2 n}\right) \\
=I_{N} \otimes A_{\mathrm{veh}}+\widetilde{L}_{G} \otimes B_{\mathrm{veh}} F_{\mathrm{veh}} .
\end{gathered}
$$

The right-hand side is block upper triangular. Its diagonal blocks are of the form:

$A_{\mathrm{veh}}+\lambda B_{\mathrm{veh}} F_{\mathrm{veh}}$,

where $\lambda$ is an eigenvalue of $L_{G}$. There is one block for each eigenvalue (counting multiplicity). Therefore, the eigenvalues of $A+B F L$ are those of $A_{\mathrm{veh}}+\lambda B_{\mathrm{veh}} F_{\mathrm{veh}}$ for $\lambda$ an eigenvalue of $L_{G}$.

We now give precise definitions of all graphtheoretic terms.

\section{Algebraic graph theory}

For our purposes, a directed graph or digraph $G$ consists of a finite set $\mathscr{V}$ of vertices and a set $\mathscr{E} \subseteq$ $V \times V$ to be referred to as (directed) edges. We will assume that the digraph has no loops, that is $(x, y) \in$ $\mathscr{E}$ implies $x \neq y$. If the digraph has the property that $(x, y) \in \mathscr{E}$ implies $(y, x) \in \mathscr{E}$ we will say that it is undirected and just call it a graph. In the vehicle application this corresponds to having bidirectional communication.

A (directed) walk in a digraph is a finite sequence of edges $\left(a_{k}, b_{k}\right) k=1, \ldots, r$ such that $b_{k}=a_{k+1}$ for $k=1, \ldots, r-1$. A walk with distinct vertices is called a (directed) path. A walk is called a (directed) circuit if in addition $b_{r}=a_{1}$. We say a digraph $G$ is strongly connected if for any vertices $i, j \in \mathscr{V}$, there exists a walk in $G$ from $i$ to $j$. Each digraph has an associated (undirected) graph obtained by adding to $E$ every pair $(y, x)$ for which $(x, y)$ is in $E$. We say that a digraph is weakly connected if the associated graph is connected, that is, if there is a walk in the associated graph connecting any two vertices.

If $G$ is strongly connected, then for any $i, j \in \mathscr{V}$, we define the distance between $i$ and $j$ to be the number of edges in a shortest walk joining $i$ and $j$. The diameter $\mathscr{D}$ of a connected graph $G$ is the maximum distance between any two vertices of $G$.

Let $G$ denote a digraph with vertex set $\mathscr{V}$ and edge set $\mathscr{E}$. Let Mat $\mathscr{N}(\mathbb{R})$ denote the set of all matrices with real entries whose rows and columns are indexed by 
the vertices of $G$. By the adjacency matrix of $G$ we mean the matrix $Q \in \operatorname{Mat}_{\mathscr{V}}(\mathbb{R})$ with entries

$q_{i j}=\left\{\begin{array}{ll}1 & \text { if }(j, i) \in \mathscr{E} \\ 0 & \text { otherwise }\end{array} \quad(i, j \in \mathscr{V})\right.$.

When $G$ is undirected, the matrix $Q$ is symmetric. The in-degree matrix of $G$ is the diagonal matrix $D \in$ $\operatorname{Mat}_{\mathscr{N}}(\mathbb{R})$ with diagonal entries

$d_{i i}=|\{j \in \mathscr{V}:(j, i) \in \mathscr{E}\}| \quad(i \in \mathscr{V})$.

The directed Laplacian of $G$ is the matrix defined by [2]

$L_{G}=D-Q$.

We use the word directed to distinguish this matrix from the most common definition of Laplacian in the graph theory literature [28] as $M_{G} M_{G}^{\mathrm{T}}$ where $M_{G}$ is the incidence matrix of $G$. This definition always results in the same symmetric matrix regardless of whether the graph is directed or not. We prefer the first definition since it is the matrix $L_{G}$ which is the most relevant to this problem. Both definitions agree for undirected graphs.

If every vertex has nonzero in-degree then $D$ is invertible. In this case an alternative definition of a Laplacian could be $\mathscr{L}=I_{N}-D^{-1} Q$ [3,4]. (If $D$ is not invertible this formula for $\mathscr{L}$ can be adapted by using pseudo-inverses [26].) These two matrices $L_{G}$ and $\mathscr{L}$ have slightly different properties which make each more advantageous than the other in different circumstances.

Some relatively simple, but powerful, results about the spectrum of $L_{G}$ are the following:

1. For a digraph with $N$ vertices, all the eigenvalues of $L_{G}$ have nonnegative real part less than or equal to $2(N-1)$ (use Gershgorin's theorem). Moreover, except for the eigenvalue zero, the real part of all other eigenvalues is positive.

2. Zero is an eigenvalue of $L_{G}$ and all the ones vector $\mathbf{1}$ is an associated eigenvector.

3. If $G$ is an undirected and connected graph on $N$ vertices, then $L_{G}$ is symmetric and each nonzero eigenvalue $\lambda$ of $L_{G}$ is real and satisfies (see [14])

$$
N \geqslant \lambda \geqslant \frac{4}{N \mathscr{D}},
$$

where $\mathscr{D}$ denotes the diameter of $G$. There is also a lower bound in terms of the edge-connectivity $e(G)$ (see [6]): $\lambda \geqslant 2 e(G)(1-\cos (\pi / N))$.

4. If $G$ is undirected then the least nonzero eigenvalue $\lambda_{1}$ of $L_{G}$ grows monotonically with the number of edges. More precisely, adding edges never decreases $\lambda_{1}[6]$.

We include below as example a list of spectra of various well known classes of digraphs. An eigenvalue $\lambda$ with multiplicity $k$ is denoted $\lambda^{k}$.

\begin{tabular}{lll}
\hline Name & \# ver. & Eigenvalues \\
\hline Complete graph $K_{n}$ & $n$ & $0, n^{n-1}$ \\
Complete bipartite & $m+n$ & $0, m^{n-1}, n^{m-1}$, \\
$K_{m, n}$ & & $m+n$ \\
Out-directed star $\mathscr{S}_{n}$ & $n$ & $0,1^{n-1}$ \\
Undirected star $S_{n}$ & $n$ & $0,1^{n-2}, n$ \\
Directed path $\mathscr{P}_{n}$ & $n$ & $0,1^{n-1}$ \\
Undirected path $P_{n}$ & $n$ & $2\left(1-\cos \left(\frac{\pi k}{n}\right)\right)$ \\
& & $k=0, \ldots, n-1$ \\
Directed cycle $\mathscr{C}_{n}$ & $n$ & $1-\mathrm{e}^{2 \pi \mathrm{i} k / n}$ \\
& & $k=0, \ldots, n-1$ \\
Undirected cycle $C_{n}$ & $n$ & $2\left(1-\cos \left(\frac{2 \pi k}{n}\right)\right)$ \\
& & $k=0, \ldots, n-1$ \\
$n$-cube $Q_{n}$ & $2^{n}$ & $(2 k)\left(\begin{array}{c}n \\
k\end{array}\right)$ \\
& & $k=0, \ldots, n$ \\
\hline
\end{tabular}

We will need a special property of the eigenvalue zero for our purposes. For that we need additional definitions.

Definition 3.1. A rooted directed tree is a digraph $T$ with the following properties:

- $T$ has no cycles.

- There exists a vertex $v$ (the root) such that there is a (directed) path from $v$ to every other vertex in $T$.

The following result is classical.

Theorem 3.2 (Tutte (1948), see [28]). Given a (loopless) digraph $G$, let $L_{G}=D-Q$, where $Q$ is the adjacency matrix and $D$ is the in-degree matrix. The number of spanning directed trees of $G$ rooted at vertex $v_{i}$ is the value of each co-factor in the ith row of $L_{G}$. 
The following result can be proved using a suitable expansion of the determinant.

Proposition 3.3. Let $M$ be a matrix and $p(x)=$ $\operatorname{det}(x I-M)$ denote its characteristic polynomial. The coefficient of $x$ in $p(x)$ is given by the sum of the principal co-factors of $M$.

Proposition 3.4. Let $G$ denote a (loopless) digraph. Then, zero is an eigenvalue of algebraic multiplicity one for the directed Laplacian $L_{G}$ if and only if $G$ has a rooted directed spanning tree.

Proof. Since zero is an eigenvalue the polynomial $p(x)$ has zero constant term. The eigenvalue 0 has algebraic multiplicity greater than 1 if and only if the coefficient of $x$ in $p(x)$ is zero. By Tutte's theorem and the previous proposition this is equivalent to having no rooted directed spanning tree.

Remark 3.5. We will show below that the stabilizing feedback law proposed in the second section exists if and only if zero has algebraic multiplicity one. This shows the exact extent of applicability of this approach.

For additional graph theoretic terms and results see [8].

\section{Stabilizability}

We show first that for the present decentralized feedback law to achieve formation stability, the individual vehicle dynamics must have a particular form. We recall that we are assuming that $A_{\text {veh }}$ and $B_{\text {veh }}$ have the form indicated in (2).

The following result was proved in [10] for the case $a_{21}^{1}=\cdots=a_{21}^{n}$ and $G$ a connected undirected graph. A similar argument applies for our more general case.

Proposition 4.1. If for every formation $h$ there exists a stabilizing feedback matrix $F=I_{N} \otimes F_{\mathrm{veh}}$ such that every solution of (4) converges to formation $h$, then $a_{21}^{i}=0$ for $i=1, \ldots, n$.

One should expect the previous result to hold whenever arbitrary formations must be achieved since in that case the accelerations should not depend on the absolute positions but only on their values relative to the other vehicles. On the other hand, dependence on velocities is possible since formations might be maintained while accelerating and decelerating as long as all vehicles have the same velocities. We will assume from now on that $a_{21}^{i}=0$ for all $i$.

Remark 4.2. The above statement implies that every odd-numbered column of the matrix $A_{\text {veh }}$ (and by extension, every such column of $I_{N} \otimes A_{\text {veh }}$ ) is zero. In particular, zero is always an eigenvalue of $A_{\mathrm{veh}}$.

Remark 4.3. If zero is an eigenvalue of $L_{G}$ of algebraic multiplicity 1 then the vehicles are in formation $h$ if and only if $L(x-h)=0$. To see this notice first that the null space of $L_{G}$ is spanned by the all one's vector $\mathbf{1}_{N}$. Therefore the null space of $L=L_{G} \otimes I_{2 n}$ is spanned by $\mathbf{1}_{N} \otimes \mathbf{e}_{j}$ where $\mathbf{e}_{j}, j=1, \ldots, 2 n$ are the standard basis vectors in $\mathbb{R}^{2 n}$. The conclusion follows from Definition 2.2.

We will now show that $G$ has a rooted directed spanning tree if and only if there exist feedback matrices as described above that achieve formation. We start with the following result which characterizes convergence to formation in terms of a spectral property of $L_{G}$. A version of this appeared in [10].

Theorem 4.4. Let $G$ be a digraph with the property that zero is an eigenvalue of the directed Laplacian $L_{G}$ of algebraic multiplicity one. Then the matrix $A_{\mathrm{veh}}+$ $\lambda B_{\text {veh }} F_{\text {veh }}$ is stable (Hurwitz) for each nonzero eigenvalue $\lambda$ of $L_{G}$ if and only if, for every $h$, every solution of (4) converges to formation $h$.

Proof. As shown earlier the eigenvalues of $A+B F L$ are those of $A_{\mathrm{veh}}+\lambda B_{\mathrm{veh}} F_{\mathrm{veh}}$ for each $\lambda$ in the spectrum of $L_{G}$.

The structure of the proof is as follows. First we expand the system to $\dot{y}=M y$ using $h_{p}$ as a new variable in a standard form. Then we show that a suitable subspace is $M$-invariant. Thirdly, we show that the map induced on the quotient space is stable if and only if $A_{\text {veh }}+\lambda B_{\text {veh }} F_{\text {veh }}$ is stable for each nonzero eigenvalue $\lambda$ of the $L_{G}$. Finally, we show that convergence in the quotient space means convergence to formation for solutions of (4). 
Since the desired formation is a constant vector, the formation variable $h_{p}$ satisfies $\dot{h}_{p}=0$. We consider the extended system

$$
\begin{aligned}
\dot{x}= & A x+B F L x \\
& -B F L\left(I_{n N} \otimes\left(\begin{array}{l}
1 \\
0
\end{array}\right)\right) h_{p},
\end{aligned}
$$

$\dot{h}_{p}=0$,

Notice that

$h=h_{p} \otimes\left(\begin{array}{l}1 \\ 0\end{array}\right)=\left(I_{n N} \otimes\left(\begin{array}{l}1 \\ 0\end{array}\right)\right) h_{p}$.

We write the above equations as $\dot{y}=M y$ where $y=$ $\left(\begin{array}{c}x \\ h_{p}\end{array}\right)$ and $M$ is the $(3 n N) \times(3 n N)$ matrix given by

$M=\left(\begin{array}{cc}A+B F L & -B F L\left(I_{n N} \otimes\left(\begin{array}{l}1 \\ 0\end{array}\right)\right) \\ \mathbf{0} & \mathbf{0}_{n N}\end{array}\right)$.

By our earlier comments about the eigenvalues of $A+B F L$, this shows that the spectrum of $M$ consists of the eigenvalues of the $n N \times n N$ matrix of zeros and those of $A_{\text {veh }}+\lambda B_{\text {veh }} F_{\text {veh }}$ for $\lambda$ in the spectrum of $L_{G}$.

Let $\mathscr{S}$ be the space having the basis

$$
\begin{aligned}
\mathbf{B}= & \left\{\left(\begin{array}{c}
\mathbf{1}_{N} \otimes \mathbf{e}_{i} \\
0
\end{array}\right): \mathbf{e}_{i} \in \mathbb{R}^{2 n}, i=1, \ldots, 2 n\right\} \\
& \cup\left\{\left(\begin{array}{c}
\left.\left.\mathbf{e}_{j} \otimes\left(\begin{array}{c}
1 \\
0
\end{array}\right)\right): \mathbf{e}_{j} \in \mathbb{R}^{n N}, j=1, \ldots, n N\right\} .
\end{array}\right.\right.
\end{aligned}
$$

Claim. The space $\mathscr{S}$ is M-invariant.

An element of $\mathscr{S}$ has the form

$y=\left(\begin{array}{c}\mathbf{1}_{N} \otimes \alpha \\ 0\end{array}\right)+\left(\begin{array}{c}\beta \otimes\left(\begin{array}{l}1 \\ 0\end{array}\right) \\ \beta\end{array}\right)$

with $\alpha \in \mathbb{R}^{2 n}$ and $\beta \in \mathbb{R}^{n N}$. From the basic rules of multiplication of Kronecker products we get that

$$
\left(I_{n N} \otimes\left(\begin{array}{l}
1 \\
0
\end{array}\right)\right) \beta=\beta \otimes\left(\begin{array}{l}
1 \\
0
\end{array}\right) .
$$

This implies that

$$
B F L\left(\beta \otimes\left(\begin{array}{l}
1 \\
0
\end{array}\right)\right)-B F L\left(I_{n N} \otimes\left(\begin{array}{l}
1 \\
0
\end{array}\right)\right) \beta=0 .
$$

Combining this with Eqs. (6) and (7), we obtain

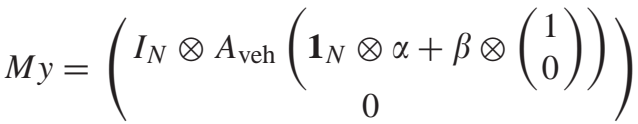

$$
\begin{aligned}
& =\left(\begin{array}{c}
\mathbf{1}_{N} \otimes A_{\mathrm{veh}} \alpha \\
0
\end{array}\right) \in \mathscr{S} .
\end{aligned}
$$

The last equality follows from Remark 4.2 and the fact that the vector $\beta \otimes\left(\begin{array}{l}1 \\ 0\end{array}\right)$ has zeros in its even numbered rows.

This calculation also shows that the matrix of the restriction of the transformation induced by $M$ on $\mathscr{S}$ relative to the basis above is exactly

$$
\left(\begin{array}{cc}
A_{\mathrm{veh}} & 0 \\
0 & \mathbf{0}_{n N}
\end{array}\right) \text {. }
$$

We now note that from the definitions of $\mathscr{S}$ and $\mathscr{F}_{h}$ we have that

$$
\begin{aligned}
& \left(\begin{array}{c}
x \\
h_{p}
\end{array}\right) \in \mathscr{S} \\
& \quad \Leftrightarrow \exists \alpha \in \mathbb{R}^{2 n}, \beta \in \mathbb{R}^{n N}: x=\mathbf{1}_{N} \otimes \alpha+\beta \otimes\left(\begin{array}{l}
1 \\
0
\end{array}\right), \\
& h_{p}=\beta \Leftrightarrow x \in \mathscr{F}_{h} .
\end{aligned}
$$

Since 0 is an eigenvalue of $L_{G}$ of (algebraic) multiplicity 1 , then the eigenvalues of $A+B F L$ are those of $A_{\text {veh }}$ together with those of $A_{\text {veh }}+\lambda B_{\text {veh }} F_{\text {veh }}$ for each nonzero $\lambda$ in the spectrum of $L_{G}$. Therefore, the matrix $M$ induces a linear transformation on the quotient space $\mathbb{R}^{2 n N} / \mathscr{S}$ whose eigenvalues are those of $A_{\text {veh }}+\lambda B_{\text {veh }} F_{\text {veh }}$ for $\lambda$ a nonzero eigenvalue of $L_{G}$.

We conclude that the quotient dynamics are stable if and only if $A_{\mathrm{veh}}+\lambda B_{\mathrm{veh}} F_{\mathrm{veh}}$ is Hurwitz for each $\lambda \neq 0$ in the spectrum of $L_{G}$. Moreover, stability of the quotient dynamics is equivalent to $y+\mathscr{S} \rightarrow \mathscr{S}$ whenever $y=\left(\begin{array}{c}x \\ h_{p}\end{array}\right)$ and $\dot{y}=M y$. From the characterization of $\mathscr{S}$ this means that each solution $x(t)$ of (4) converges to formation.

We now want to show that stabilizing feedback matrices indeed exist. For this we need some preliminary calculations expressing the eigenvalues of $A_{\mathrm{veh}}+$ $\lambda B_{\text {veh }} F_{\text {veh }}$ in terms of $\lambda$. It will suffice if we can find feedback matrices of the form $F_{\text {veh }}=I_{n} \otimes\left(f_{1}, f_{2}\right)$. We use Routh's criterion to develop conditions on the feedback coefficients (gains) $f_{1}, f_{2}$ to guarantee convergence to formation. 
Let $q(x)=x^{2}+s x+p$, be a polynomial where $s=s_{1}+s_{2} i$ and $p=p_{1}+p_{2} i$ are complex numbers. If $\mu$ is a root of $q(x)$ then $\bar{\mu}$ is a root of $r(x)=x^{2}+$ $\bar{s} x+\bar{p}$. Therefore $q(x)$ is stable if and only if $h(x)=$ $q(x) r(x)$ is. Applying Routh's criterion to $h(x)$ (which has real coefficients) results in the following necessary and sufficient conditions for stability of $q(x)$ :

$s_{1}>0 \quad 2 p_{1}+s_{1}^{2}+s_{2}^{2}>0$,

$s_{1} p_{1}+s_{2} p_{2}>0, \quad p_{1} s_{1}^{2}+p_{2} s_{1} s_{2}-p_{2}^{2}>0$.

We apply these conditions to the specific case at hand. We are interested in conditions on the real scalars $f_{1}$ and $f_{2}$ that would make the characteristic polynomial of $A+\lambda B F$ have roots with negative real part.

As before, for the entire system let $u=F L(x-h)$, where $F=I_{n N} \otimes\left(f_{1}, f_{2}\right)$ and $L=L_{G} \otimes I_{n}$. The stability problem reduces to finding $f_{1}$ and $f_{2}$ such that $A+\lambda B F$ is Hurwitz for each nonzero eigenvalue $\lambda$ of $L_{G}$. Notice that those eigenvalues have positive real part (see Section 3). To simplify the notation we assume further that each coordinate has the exact same dynamic equations. (The general case can be treated similarly with one set of inequalities for each coordinate.) We get

$$
\begin{aligned}
A+\lambda B F & =I_{n N} \otimes\left(\left(\begin{array}{cc}
0 & 1 \\
0 & a_{22}
\end{array}\right)+\lambda\left(\begin{array}{l}
0 \\
1
\end{array}\right)\left(f_{1}, f_{2}\right)\right) \\
& =I_{n N} \otimes\left(\begin{array}{cc}
0 & 1 \\
\lambda f_{1} & a_{22}+\lambda f_{2}
\end{array}\right) .
\end{aligned}
$$

The eigenvalues of $A+\lambda B F$ are the roots of polynomial $q(x)=x^{2}+\left(-a_{22}-\lambda f_{2}\right) x+\left(-\lambda f_{1}\right)$. Writing $\lambda=\alpha+\beta i$ and using the above formulas we obtain the following necessary and sufficient conditions for the polynomial to be stable:

$$
\begin{aligned}
& -a_{22}-\alpha f_{2}>0, \\
& -2 \alpha f_{1}+\left(a_{22}+\alpha f_{2}\right)^{2}+\beta^{2} f_{2}^{2}>0, \\
& a_{22} \alpha f_{1}+\left(\alpha^{2}+\beta^{2}\right) f_{1} f_{2}>0, \\
& -\alpha f_{1}\left(a_{22}+\alpha f_{2}\right)^{2}-\beta^{2} f_{1} f_{2}\left(a_{22}+\alpha f_{2}\right) \\
& \quad-\beta^{2} f_{1}^{2}>0 .
\end{aligned}
$$

Since $\alpha>0$, looking at the signs of the coefficients of $f_{1}$ and $f_{2}$ it is easy to see that all inequalities are satisfied by choosing $f_{1}<0, f_{2}<0$ and $f_{2}$ large enough in absolute value. We have shown the following.

Proposition 4.5. The gains $f_{1}, f_{2}$ stabilize the formation if and only if they satisfy the four inequalities above. Moreover, the system of inequalities always has solutions. More specifically, one such stabilizing pair can be found by choosing $f_{1}<0$ and $f_{2}<0$ with $f_{2}$ sufficiently large in absolute value.

We can make this more explicit in the case $a_{22}=0$, which corresponds to each coordinate being modelled as a double integrator. The conditions become simply:

$f_{1}<0$,

$f_{2}<0$,

$\frac{f_{2}^{2}}{f_{1}}<-\frac{\beta^{2}}{\alpha\left(\alpha^{2}+\beta^{2}\right)}$,

which can be easily satisfied for some $f_{1}, f_{2}$.

We now present the main results of the paper.

Theorem 4.6. Consider the control system given by (4). There exists a matrix $F_{\text {veh }}$ such that for every formation $h$ the solution to (4) converges to formation $h$ if and only if zero has multiplicity one as an eigenvalue of the directed graph Laplacian $L_{G}$.

Proof. The sufficiency follows from Theorem 4.4 and Proposition 4.5.

For the necessity assume that zero is an eigenvalue of $L_{G}$ of multiplicity $k>1$. We proceed as in the proof of Theorem 4.4 to construct the matrix $M$, the basis B, and the space $\mathscr{S}$. The difference is that now $\mathscr{S}$ is a proper subset of

$\left\{\left(\begin{array}{c}x \\ h_{p}\end{array}\right): L(x-h)=0\right\}$.

Convergence to formation is still equivalent to convergence in the quotient space. However, regardless of $F_{\text {veh }}$ the spectrum of the quotient map now includes at least $k-1$ copies of the eigenvalues of $A_{\text {veh }}$ one of which is zero (see Remark 4.2). This implies that the quotient dynamics cannot be stabilized with the above type of feedback laws. 
Combining Proposition 3.4 with the previous theorem gives the following.

Corollary 4.7. Consider the control system given by (4). There exists a matrix $F_{\text {veh }}$ such that for every formation $h$ the solution to (4) converges to formation $h$ if and only if the digraph $G$ has a rooted directed spanning tree.

\section{Examples}

We illustrate the main results with several numerical examples. First we assume that $a_{22}=0$ so each coordinate is modelled as a double integrator. In all these examples the desired formation is specified as the vertices of a regular pentagon. The initial position of the vehicles is marked with an ' $x$ ' and all vehicles are lined up in a row at the start. The final positions of the vehicles are marked with circles. Fig. 2 shows convergence to formation using the same feedback matrix but three different digraphs, the (directed) 5-cycle, the (directed) 5-path, and the complete graph $K_{5}$. The formation drifts in space at a constant speed because vehicle 1 has an initial nonzero velocity. Notice that while the cycle has one edge more than the path, the former achieves formation more slowly than the latter. The corresponding (approximate) spectra of $A+B F L$ are (without counting multiplicities): $\{0,-0.22 \pm 1.88 \mathrm{i},-0.81 \pm$ $0.45 \mathrm{i},-1.21 \pm 0.60 \mathrm{i},-1.51 \pm 1.48 \mathrm{i}\}$ for the cycle, $\{0,-0.75 \pm 0.97 \mathrm{i}\}$ for the path, and $\{0,-1.19,-6.31\}$ for $K_{5}$. We will see below that for undirected graphs convergence cannot deteriorate when more edges are added.

In Fig. 3 the same communication digraph is used (the directed cycle) with different feedback matrices.

\section{Special cases}

We discuss here two categories of graphs which have special interest in applications.

\subsection{Bidirectional communications}

If communication is bidirectional, then whenever vehicle $i$ receives information from vehicle $j$, vehicle $j$ also receives information from vehicle $i$. This means that the adjacency matrix of the communication graph is symmetric and so all its eigenvalues are real.

The discriminant of the characteristic polynomial of the matrix $A_{\text {veh }}+\lambda B_{\text {veh }} F_{\text {veh }}$ is $\left(a_{22}+\lambda f_{2}\right)^{2}+4 \lambda f_{1}$. Thus, for a fixed $f_{2}$, choosing $f_{1}$ so that

$$
\frac{\left(a_{22}+\lambda f_{2}\right)^{2}}{4 \lambda}<-f_{1}
$$

for every nonzero eigenvalue $\lambda$ of $L_{G}$ guarantees complex (nonreal) roots of the characteristic polynomial, thereby providing the fastest rate of convergence. Thus we have proved the following.

Proposition 6.1. Assume $G$ is undirected. For $f_{1}$ and $f_{2}$ as above, the rate of convergence to formation is $\left(a_{22}+\lambda_{1} f_{2}\right) / 2$, where $\lambda_{1}$ is the smallest nonzero eigenvalue of $L_{G}$.

In this case, where the graphs are undirected, the monotonicity property of $\lambda_{1}$ (see Section 3 ) and the above inequality show that if $f_{2}$ is fixed the convergence to formation cannot deteriorate by adding edges.

Remark 6.2. For a fixed number $N$ of vehicles, stabilizing gains $f_{1}$ and $f_{2}$ can be chosen independently of the graph. This follows immediately from the inequalities (5).

\subsection{Formations with leaders}

A special situation occurs when one of the vehicles does not receive information from any of the others. Essentially this means that the others are forced to arrange their positions in response to the motion of that vehicle. This makes such a vehicle a de facto leader. The motion of the overall formation is dictated by that of the leader. Since we are always assuming that the communication digraph has a directed rooted spanning tree, there can be at most one such leader. Since the in-degree of the corresponding vertex is zero, the corresponding row of the adjacency matrix is zero and the matrix $D$ is not invertible.

In the following figures the initial position of the vehicles is marked with an " $x$ " and the desired formation consists of the vertices of a regular pentagon. The leader is marked with a diamond. The communication digraph is a directed path. In Fig. 4 the leader 

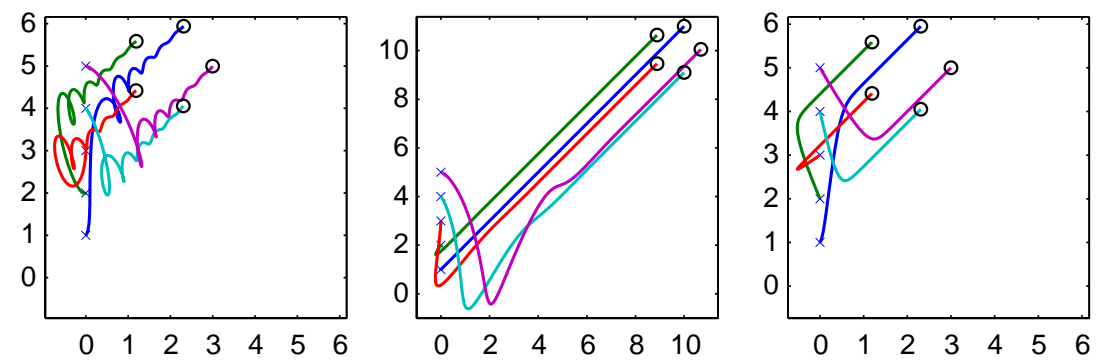

Fig. 2. Form left: (directed) cycle, (directed) path, and complete graph.
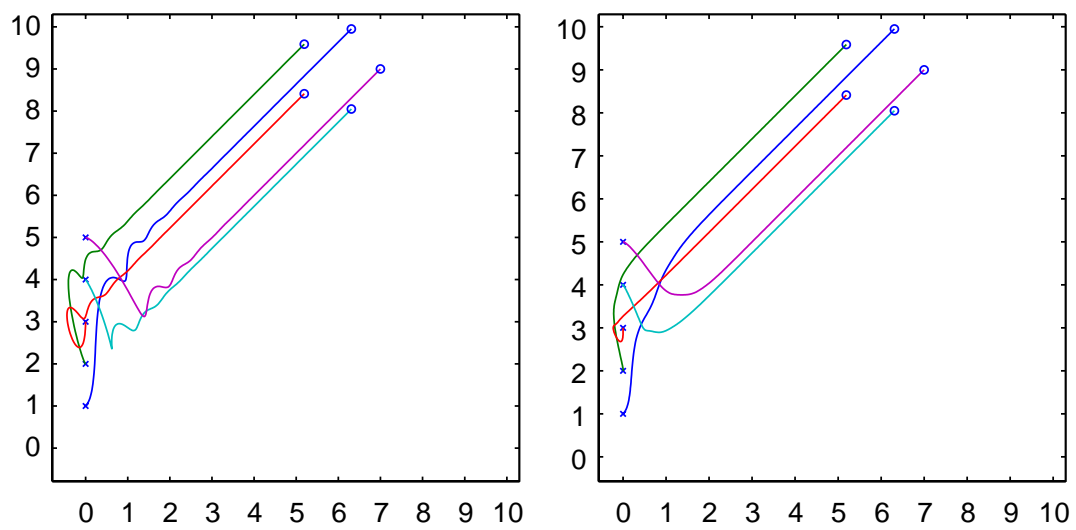

Fig. 3. Cycle with different feedback gains: $f_{1}$ and $f_{2}$ are more negative on the right.
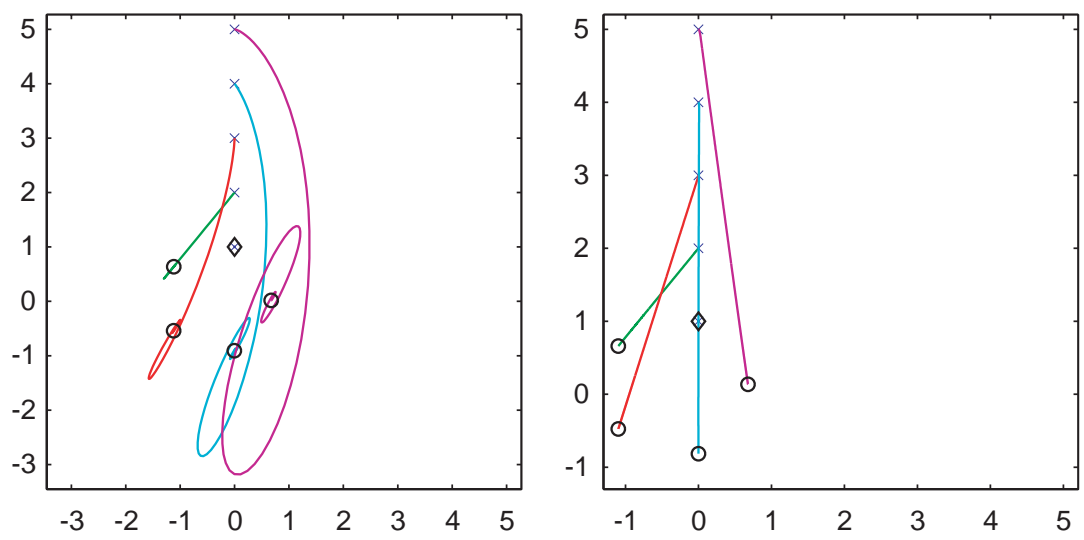

Fig. 4. Formations with leaders. Stronger feedback laws from left to right. The leader is indicated with a diamond.

has zero initial velocities and therefore remains fixed in its position. In Fig. 5 the leader has an initial velocity (in the direction $(1,1)$ ) and therefore the whole formation drifts in that direction. Notice how the various vehicles have to adjust their own path to achieve the formation. 

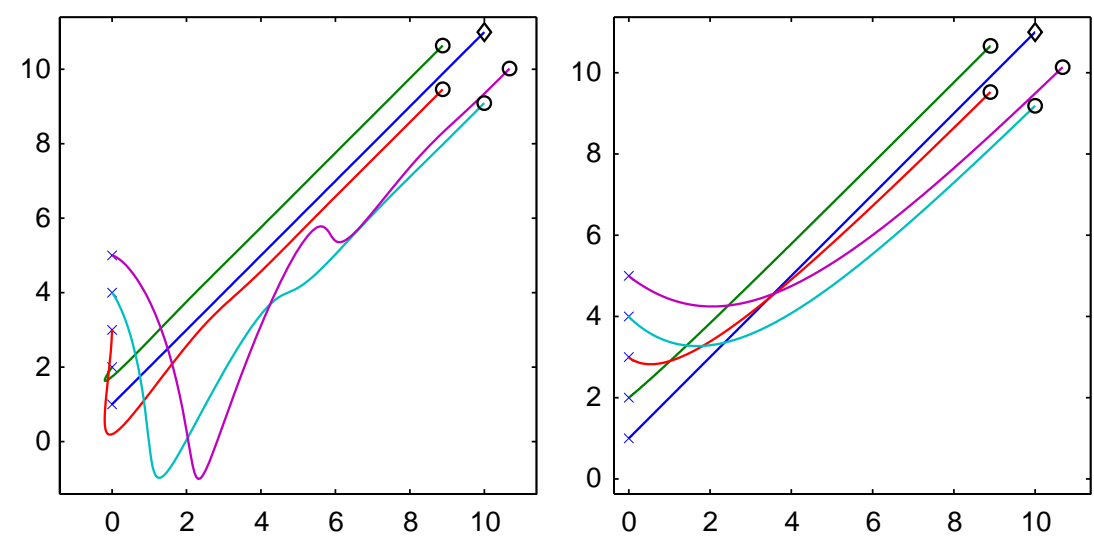

Fig. 5. Formations with leaders. Leader drifts due to initial velocity. The leader is indicated with a diamond.
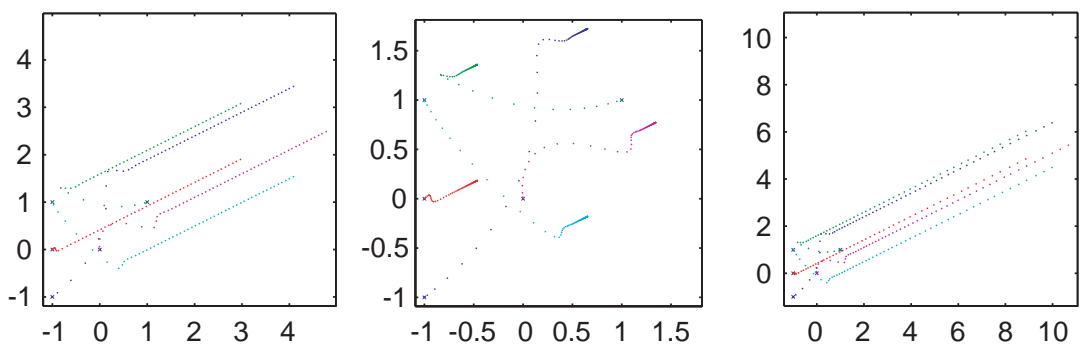

Fig. 6. Effect of $a_{22}$. From left to right $a_{22}=0, a_{22}<0, a_{22}>0$.

\section{Moving in formation}

Fig. 6 illustrates the effect of the $a_{22}$ term in the resulting formations. While these examples use the same $2 \times 2$ matrix for each controlled quantity in a single vehicle, the effect of different values for each of them should be clear from these pictures. The dots are plotted at equal time intervals. The model still assumes that all the vehicles have the same dynamics. For $a_{22}=0$ the vehicles achieve a constant velocity. For $a_{22}<0$ the vehicles eventually stop. For $a_{22}>0$ the vehicles uniformly accelerate.

\section{Concluding remarks}

We have demonstrated the close connection between spectral graph theory and one of the current methods of control of vehicle formations. We have made explicit how to choose stabilizing feedback gains in terms of the eigenvalues of the directed Laplacian of the communication digraph. Furthermore, for undirected graphs we have derived an expression for the rate of convergence to formation that is a linear function of the smallest positive eigenvalue of the Laplacian.

We have used throughout the Laplacian $D-Q$. A similar approach could be applied to the Laplacian $I-D^{-1} Q$ (when $D$ is invertible). The difference is that the matrix $D$, in effect, produces different scalings for the different vehicles. In the case of in-regular digraphs, the matrix $D$ is a scalar multiple of $I$ and the resulting gains are equivalent. The question of finding bounds for $\lambda_{1}$ when $G$ is undirected is well studied. For the directed case we find that (for $a_{22}=0$ ) the quantity $\beta^{2} /\left[\alpha\left(\alpha^{2}+\beta^{2}\right)\right]$ is the key indicator of stability margins.

Some generalizations seem possible. We expect that using a weighted communication digraph will equally allow for convergence to formation. The different weights will cause the net effect of the feedback laws to be different for each vehicle. A more significant generalization would be to allow the digraph to change. The techniques from [9] suggest a way 
to deal with the problem although the flexibility in the choice of formations and the use of more general Laplacians complicate the convergence studies.

\section{Acknowledgements}

The authors wish to thank the reviewers for pointing out several references and for their many thoughtful comments.

\section{References}

[1] G. Ayres de Castro, F. Paganini, Convex synthesis of controllers for consensus, in: Proceedings of the American Control Conference, June 2004, pp. 4933-4938.

[2] R. Brualdi, H. Ryser, Combinatorial Matrix Theory, vol. 39 of Encyclopedia of Mathematics and Its Applications, Cambridge University Press, Cambridge, 1991.

[3] F.R.K. Chung, Spectral Graph Theory, CBMS Regional Conference Series in Mathematics, American Mathematical Society, Providence, RI, 1997.

[4] J.A. Fax, Optimal and cooperative control of vehicle formations, Ph.D. Thesis, California Institute of Technology, 2001.

[5] J.A. Fax, R.M. Murray, Information flow and cooperative control of vehicle formations, IEEE Trans. Automat. Control 49 (9) (2003) 1465-1476.

[6] M. Fiedler, Algebraic connectivity of graphs, Czechoslovak Math. J. 23 (98) (1973) 298-305.

[7] S. Glavaski, M. Chavez, R. Day, L. Gomez-Ramos, P. Nag, A. Williams, W. Zhang, Vehicle networks: achieving regular formation, IMA Tech. Rep., 2002.

[8] C.D. Godsil, Algebraic Combinatorics, Chapman \& Hall, New York, 1993.

[9] A. Jadbabaie, J. Lin, A.S. Morse, Coordination of groups of mobile autonomous agents using nearest neighbor rules, IEEE Trans. Automat. Control 48 (6) (2003) 998-1001.

[10] G. Lafferriere, J. Caughman, A. Williams, Graph theoretic methods in the stability of vehicle formations, ACC2004, July 2004, pp. 3724-3729.

[11] J. Lawton, R. Beard, B. Young, A decentralized approach to formation maneuvers, IEEE Trans. Robotics Automat. 19 (6) (2003) 933-941.

[12] N.E. Leonard, E. Fiorelli, Virtual leaders, artificial potentials and coordinated control of groups, in: Proceedings of IEEE Conference on Decision and Control, 2001, pp. 2968-2973.
[13] N.E. Leonard, P. Ogren, Obstacle avoidance in formation, IEEE ICRA, September 2003, pp. 2492-2497.

[14] R. Merris, Laplacian graph eigenvectors, Linear Algebra Appl. 278 (1998) 221-236.

[15] L. Moreau, Leaderless coordination via bidirectional and unidirectional time-dependent communication, in: Proceedings of IEEE Conference on Decision and Control, December 2003, pp. 3070-3075.

[16] A. Okubo, Dynamical aspects of animal grouping: swarms, schools, flocks and herds, Adv. Biophys. 22 (1986) 1-94.

[17] R. Olfati-Saber, R. Murray, Agreement problems in networks with directed graphs and switching topology, in: Proceedings of IEEE Conference on Decision and Control, December 2003, pp. 4126-4132.

[18] R. Olfati-Saber, R. Murray, Consensus protocols for networks of dynamic agents, in: Proceedings of the American Control Conference, July 2003, pp. 951-956.

[19] W. Ren, R. Beard, Consensus of information under dynamically changing interaction topologies, in: Proceedings of the American Control Conference, June 2004, pp. 4939-4944.

[20] W. Ren, R. Beard, A decentralized scheme for spacecraft formation flying via the virtual structure approach, AIAA J. Guidance, Control Dynamics 27 (1) (2004) 73-82.

[21] W. Ren, R. Beard, Consensus seeking in multi-agent systems using dynamically changing interaction topologies, IEEE Trans. Automat. Control, to appear.

[22] A.G. Sparks, Special issue on control of satellite formations, Internat. J. Robust Nonlinear Control 12 (2-3) (2002).

[23] H. Tanner, A. Jadbabaie, G. Pappas, Stable flocking of mobile agents, part I: fixed topology, in: Proceedings of IEEE Conference on Decision and Control, December 2003, pp. 2010-2015.

[24] H. Tanner, A. Jadbabaie, G. Pappas, Stable flocking of mobile agents, part II: dynamic topology, in: Proceedings of IEEE Conference on Decision and Control, December 2003, pp. 2016-2021.

[25] H.G. Tanner, A. Jadbabaie, G.J. Pappas, Flocking in fixed and switching networks, Automatica, submitted for publication.

[26] J.J.P. Veerman, G. Lafferriere, J.S. Caughman, A. Williams, Flocks and formations, J. Statist. Phys., submitted. Available at http://www.mth.pdx.edu/ veerman/flocks2.pdf.

[27] T. Vicsek, A. Czirok, E. Ben Jacob, I. Cohen, O. Schochet, Novel type of phase transitions in a system of self-driven particles, Phys. Rev. Lett. 75 (1995) 1226-1229.

[28] D.B. West, Introduction to Graph Theory, Prentice-Hall, London, 2001.

[29] A. Williams, S. Glavăski, T. Samad, Formations of formations: hierarchy and stability, ACC2004, July 2004, pp. 2992-2997. 